\section{PSICOLOGÍA IBEROAMERICANA}

\section{Psicología lberoamericana}

ISSN: 1405-0943

psicología.iberoamericana@uia.mx

Universidad Iberoamericana, Ciudad de

México

México

Quezadas Barahona, Ana Luisa; Pérez-Castro, Judith Análisis exploratorio de un modelo para el manejo de desastres en niños tabasqueños Psicología Iberoamericana, vol. 20, núm. 2, julio-diciembre, 2012, pp. 66-73

Universidad Iberoamericana, Ciudad de México

Distrito Federal, México

Disponible en: http://www.redalyc.org/articulo.oa?id=133928816009

Cómo citar el artículo

Número completo

- Más información del artículo

Página de la revista en redalyc.org

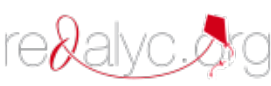

Sistema de Información Científica

Red de Revistas Científicas de América Latina, el Caribe, España y Portugal Proyecto académico sin fines de lucro, desarrollado bajo la iniciativa de acceso abierto 


\title{
Análisis exploratorio de un modelo para el manejo de desastres en niños tabasqueños
}

\author{
The Development of a Disaster Management Model Directed to Tabascan \\ Children: An Exploratory Analysis
}

\author{
Ana Luisa Quezadas Barahona* \\ Universidad Autónoma del Estado de Hidalgo \\ Judith Pérez-Castro** \\ Universidad JuÁrez Autónoma de TABAsco
}

\section{RESUMEN}

En este artículo se exponen los resultados y se valoran los alcances de una investigación exploratoria cuyo objetivo fue diseñar un modelo de recuperación psicoafectiva para situaciones de desastre, basado en la propuesta del Fondo de las Naciones Unidas para la Infancia (UNICEF). El trabajo consistió en un estudio de caso, construido a partir de un muestreo aleatorio, desarrollado con nueve niños y niñas de la ranchería Tierra Amarilla, tercera sección, del estado de Tabasco. Además del taller sobre el manejo de desastres, se aplicó un pre-test y un post-test para ver si efectivamente había algún cambio en el comportamiento de los infantes. Algunos de los avances registrados fueron el manejo de las medidas de prevención, la ampliación de la perspectiva sobre los desastres y los aprendizajes que se pueden obtener de ellos. Sin embargo, en aspectos como las habilidades de resiliencia y los valores se observaron pocos cambios.

Palabras clave: atención psicológica, resiliencia, prevención de desastres, niños.

\section{ABSTRACT}

In this paper, we discuss the results of an exploratory research whose objective was to design a psychological model to cope with disasters, based on the proposal made by the United Nations Children's Fund (UNICEF), and to assess its effects. Mainly, it was a case study, made up by a random sampling, developed with nine children from a Tabascan village called Tierra Amarilla, third section. Along with the workshop on disasters management, we implemented a pre-test and a posttest in order to estimate the changes in the children's psycho-emotional behavior. The participants showed some progresses, talking about the preventive measures. They also learnt about the different kinds of disasters, discussed about their own experiences and about the things they could learn after a disaster. However, at the same time, we noticed very few advances regarding their abilities of resilience and their values.

Keywords: psychological assistance, resilience, disaster prevention, children.

\footnotetext{
* Dirección postal: Av. Universidad s/n, Col. Magisterial. Villahermosa, Tabasco. C.P. 86040. Tel. (993) 314-31-41. Fax (993) 314-31-41 annyquezadas@hotmail.com

** Dirección postal: Av. Universidad s/n, Col. Magisterial. Villahermosa, Tabasco. C.P. 86040. Tel. (993) 314-31-41. Fax (993) 314-31-41. pkjudith33@yahoo.com.mx
} 


\section{INTRODUCCIÓN}

En las últimas dos décadas, Tabasco ha sufrido por lo menos siete inundaciones de mediana y gran magnitud. En términos económicos, los impactos más severos se han registrado en el 2007, 2008 y 2010. (Comisión Económica para América Latina y el Caribe, 2012). Pero las pérdidas han alcanzado los diferentes ámbitos: político, social, educativo y personal, además de que han evidenciado la necesidad de preparar a las personas para enfrentar futuras catástrofes y manejar sus posibles consecuencias.

La investigación cuyos resultados aquí se presentan, retoma esto último y busca atender a la población infantil a través de un modelo de recuperación psicoafectiva, con base en la propuesta hecha por el Fondo de las Naciones Unidas para la Infancia (UNICEF) (2007, 2008b y 2010). Abordar este grupo se justifica no sólo porque constituye uno de los más vulnerables, junto con las personas con capacidades especiales y las de la tercera edad (Robles \& Medina, 2002), sino también porque los niños y adolescentes son los que mayor atención psicológica han requerido a partir de los fenómenos hidrometeorológicos, alrededor del 59\% del total de consultas brindadas por el Sector Salud (CEPAL, 2011). Además, se ha reportado que debido a la capacidad de respuesta de las instituciones públicas del estado, ciertos sectores de la población no han tenido ningún acompañamiento psicológico, lo que aumenta la probabilidad de ocurrencia de trastornos emocionales, conductuales y otras alteraciones (CEPAL, 2012).

Paralelamente, existe un vasto trabajo en torno a la atención de la salud mental en escenarios de riesgo $\mathrm{y}$ de desastre que puede ser retomado para desarrollar estrategias que respondan a las necesidades de la población infantil tabasqueña. Uno de los estudios más importantes es el de Taylor (1989). En él se plantea un método de intervención enfocado a las víctimas. Se distinguen seis tipos, de las primarias a las sextarias, cuyas necesidades pueden ir desde el manejo de los estados de shock y la expresión de sus emociones o sentimientos, hasta la formación para el apoyo emocional hacia otras personas y el mantenimiento del orden.

Por su parte, Gerrity y Flinn (2000) analizan los factores que incrementan la vulnerabilidad psicológica, como: la proximidad y el tiempo de exposición al desastre, la falta de apoyo familiar, comunitario o social y la victimización secundaria. Aquí se destaca el riesgo en el que se encuentran los infantes debido a que muchos de los aspectos fundamentales para sus vidas, a saber, el hogar, sus relaciones interpersonales y contextos más próximos, tienden a verse afectados. En el mismo tenor que Taylor, estos autores señalan la importancia de realizar un diagnóstico antes de ofrecer cualquier tipo de asistencia, pues no todas las víctimas generarán una psicopatología.

Uno de los estudios más representativos sobre la atención psicológica a menores es el de Quiroz (2005), La recuperación psicoafectiva de niños afectados por desastres y conflictos armados, que parte de cinco grandes ejes: 1) las necesidades básicas y superiores de los niños, 2) la democratización del conocimiento, 3) la sinergia en la terapia auto-ayuda, 4) la familia como centro de socialización terapéutico básico y 5) la recuperación de las formas tradicionales. Propone una metodología para la recuperación psicoafectiva que parte de la base de la estrategia de atención primaria a la salud (APS) del UNICEF. El fin último es fortalecer la autoestima de los infantes, estimularlos a que expresen sus sentimientos y ayudarlos a encontrar el sentido de sus experiencias derivadas del desastre.

Otro aporte es el manual-guía de Palacio (2000), en el que se abordan diferentes cuestiones de la salud, en general y de la salud mental, en particular. Su población objetivo son los infantes por debajo de los cinco años que no han tenido suficiente apoyo social y familiar. En este contexto, la resiliencia se considera fundamental para el desarrollo de los factores protectores que ayudarán a los niños a superar el trauma y a lograr su bienestar, mientras que la escuela representa el medio principal para poner en marcha la estrategia de intervención. Sin embargo, esta propuesta se concentra en hacer una detallada descripción de los elementos del modelo y de las actividades a desarrollar en condiciones de crisis, de modo que, al final, los ejes orientadores para la promoción y prevención de la salud mental quedan un tanto desdibujados.

En este mismo enfoque de cuidado a la salud mental se encuentra la investigación de Santiesteban, Castro, González y Sánchez (2010), sólo que, en esta ocasión, las unidades de observación son un grupo de 10 niños y niñas en edad escolar que sufrieron los 
efectos de los huracanes Gustav e Ike. Aquí se destaca la utilización de técnicas proyectivas para el seguimiento de ciertos trastornos emocionales y del comportamiento. Se plantean, además, tres categorías de análisis psicológico: destrucción, afección emocional y recuperación. La principal debilidad es el número de personas que se abarcó, pero los autores concluyen que a pesar de esto el estudio les permitió observar la permanencia que pueden tener los problemas psicológicos en los niños, porque hubo la oportunidad de hacer un seguimiento a mediano plazo.

Por su parte, Tanner, Rodríguez y Lazcano (2008) intentan ampliar esta noción que se tiene de los niños y jóvenes como sujetos altamente vulnerables y necesitados de protección, para proponer un cambio de perspectiva en la que se les considera como agentes para la reducción de los riesgos y desastres. Tomando dos experiencias con grupos juveniles, los autores distinguen cuatro fases de participación: la pasiva, la endógena, la exógena y la integrada, y sostienen que los niños y jóvenes poseen la capacidad para colaborar en la reconstrucción de sus comunidades, lo que, al mismo tiempo, fortalece su identidad y puede actuar como un factor protector de problemas psicosociales. No obstante, en este caso, sólo se plantean ideas muy generales de hacia dónde podrían ir dirigidas las acciones, sin llegar a la discusión de las implicaciones de cada una de las etapas, o bien, de la forma en que la participación de los niños y jóvenes podría obstaculizar o verse obstaculizada por las acciones de otros grupos.

A la par de estas investigaciones se encuentran los reportes realizados por el Fondo de las Naciones Unidas para la Infancia (2003, 2005, 2008a, 2008b y 2009) y la organización Save the Children $(2002,2006)$, en donde se presentan resultados de estrategias y modelos de intervención implementados generalmente en condiciones de desastre y posdesastre. En estas propuestas se recuperan los aportes hechos desde la atención psicológica, en general, para adecuarla a las características y necesidades de la población infantil.

Así, el propósito de este artículo es presentar y valorar los alcances de un modelo de recuperación psicoafectiva aplicado a un grupo de infantes en situación de desastre. Se retoman conceptos y categorías generados en esta área de la investigación psicológica y también elementos de la labor realizada por las orga- nizaciones internacionales dedicadas al cuidado de los derechos, la integridad y la salud de los niños.

\section{LOS RIESGOS Y DESASTRES EN LA POBLACIÓN INFANTIL}

Un desastre es el resultado del proceso de riesgo y se caracteriza porque implica la interrupción de las actividades de una comunidad o sociedad, provoca pérdidas materiales, económicas y/o humanas y, por lo general, rebasa la capacidad de respuesta de los individuos o grupos afectados (United Nations Environment Program, 2007).

A nivel psicológico, los desastres tienen varias consecuencias, desde la negación y los estados de shock, hasta el fortalecimiento de ciertos rasgos de la personalidad. En concreto, en los infantes, los comportamientos tienden a diferenciarse de acuerdo a la edad. Según Caswell (2009), los síntomas más comunes que registran los niños entre uno y cinco años son: la ansiedad, el temor a separarse de los padres, las alteraciones en el sueño, la falta o el exceso de apetito, el miedo a animales o figuras fantásticas, el aislamiento o la poca expresividad de sus miedos o sentimientos, la zozobra ante símbolos o palabras que les recuerdan el siniestro, la recurrencia a ciertos juegos en los que se intentan recrear el desastre y algunos retrocesos en su desarrollo.

Los menores entre los seis y los 11 años pueden tener conductas muy cercanas a las ya mencionadas, pero, además tienden a presentar sentimientos de culpabilidad, desórdenes de identidad, comportamientos autodestructivos, actitudes de rebeldía y tener manifestaciones somáticas (Caswell, 2009). En la mayoría de los casos, sin importar la edad y al igual que sucede con los adultos, se puede sufrir trastorno de estrés postraumático, con la dificultad de que en ocasiones los síntomas son menos perceptibles.

Ante la ocurrencia de un siniestro, lo más importante es cuidar la integridad física y psicológica de los infantes. Sobre todo, las acciones deben orientarse a propiciar contextos y habilidades de resiliencia.

En la psicología, el término resiliencia explica la situación de aquellas personas que logran mantenerse emocionalmente sanas y tener trayectorias de vidas exitosas, a pesar de haber crecido bajo condiciones desfavorables (Uriarte, 2005). La resiliencia tiene dos com- 
ponentes: la resistencia a la adversidad, esto es, conservar la integridad, y la capacidad para restablecerse positivamente, no obstante los traumas o problemas que se hayan enfrentado (Uriarte, 2005).

En el caso de los niños y niñas, Muñoz y de Pedro (2005) retoman el modelo de Gotteberg y sostienen que para proteger a los infantes y promover en ellos actitudes resilientes es necesario trabajar en tres niveles: el ambiente social, los recursos personales y las habilidades sociales. Sin embargo, en la población infantil y adolescente, los estudios de resiliencia se han enfocado principalmente en el maltrato. Los organismos internacionales son los que más han trabajado este tema en relación con los desastres, como parte de su estrategia general para propiciar una cultura del cuidado del medio, la prevención y el manejo de riesgos. Concretamente, en esta investigación, se recurrió al Modelo de Recuperación Psicoafectiva del UNICEF (2007), el cual forma parte de un programa más amplio denominado "El retorno a la alegría". Su objetivo es mejorar la salud emocional de los menores que han pasado por situaciones de emergencia y desastre, a través de la construcción de ambientes resilientes. Se trata de una intervención en donde se utilizan actividades lúdicas y artísticas para que los niños canalicen sus emociones, expresen sus temores, rememoren sus experiencias y superen los traumas que les pudo haber producido el evento catastrófico.

El modelo contiene tres ejes: la atención primaria en salud mental, las actitudes y el desarrollo del infante. Algunos de los temas que se abordan son: el desplazamiento, el duelo, la ansiedad, la autoestima, el empoderamiento familiar, el manejo de la agresividad y la ira, la conciencia y el valor de la vida, la comunicación y los valores (UNICEF, 2007).

La recomendación es que se utilice una vez que se hayan restablecido los servicios básicos de la comunidad y que primero se implemente en la educación no formal y después en la formal, es decir, en las instituciones educativas (UNICEF, 2010). Por último, el UNICEF enfatiza que los protocolos son únicamente guías para orientar a los responsables de la intervención, pero que siempre será necesario hacer adecuaciones a medida que se vayan evidenciando los problemas y la sintomatología de los niños y niñas.

\section{MÉTODO}

El objetivo de la investigación fue diseñar y poner en marcha un modelo de intervención para el desarrollo de las habilidades de afrontamiento de desastres en estudiantes de educación primaria, con base en las características y demandas que esta población señalaba como prioritarias. Se trata de un estudio caso intrínseco en el que se trabajó con un grupo de nueve menores. Se partió del supuesto de que la población infantil es uno de los grupos más vulnerables ante la ocurrencia de un desastre y, hasta ahora, en el estado de Tabasco, ésta no ha sido tratada eficientemente. De esta manera, un modelo de intervención que atienda sus necesidades psicoafectivas y cognitivas podría mejorar sus habilidades de afrontamiento y fortalecer sus conocimientos preventivos sobre riesgos y desastres.

\section{Participantes}

Los sujetos de estudio fueron menores de la ranchería Tierra Amarilla tercera sección del municipio del Centro, Tabasco. Para seleccionarlos, y de acuerdo con la metodología del UNICEF (2007), en un primer momento se hizo una visita a la institución educativa de la comunidad con el fin de recabar la información necesaria sobre los niños. En una segunda etapa se organizó un grupo pequeño de nueve infantes. Finalmente, como tercer momento, se efectuó la intervención que constó de cinco sesiones.

En cuanto a los rasgos sociodemográficos de los participantes, cabe señalar que cinco eran del sexo femenino y cuatro del masculino; tres habían cumplido los 10 años, dos tenían nueve, dos más estaban en los ocho años y los dos últimos tenían siete. Asimismo, tres de ellos cursaban el quinto grado de primaria, dos estaban en cuarto, tres más en tercero y uno estudiaba el segundo grado.

Seis niños presentaban problemas previos en su núcleo familiar. Dos vivían separados de sus padres, bajo la tutela de sus abuelos, y cuatro provenían de familias monoparentales. Adicionalmente, se observó que estos mismos niños sufrían de violencia verbal y física en sus hogares y problemas asociados con el consumo del alcohol.

Los nueve menores provenían de familias con bajos niveles de ingreso, con padres o tutores que se dedicaban a actividades primarias, como la agricultu- 
ra y la cría de animales de traspatio. Uno de sus principales apoyos económicos era el subsidio recibido por el Programa Oportunidades de la Secretaría de Desarrollo Social (Sedesol).

A excepción de un caso, sus viviendas eran muy precarias, con piso de tierra, techos de lámina, muros de madera o de otros materiales orgánicos como la palma. La mayoría también estaban ubicadas en asentamientos irregulares, carentes de agua potable, drenaje y alcantarillado. El único servicio con el contaban regularmente era la energía eléctrica.

Con respecto a los rasgos psicológicos identificados en el diagnóstico hecho antes de la intervención, los participantes mostraban problemas de conducta: dos presentaban alteraciones en el sueño, siete tenían hiperactividad y todos manifestaban cambios en sus hábitos alimenticios. En cuanto a los cambios emocionales, se observaron altos niveles de ansiedad en la mayoría de los casos y en tres de ellos había rebeldía y actitudes de desafío a la autoridad. En general, las niñas tendieron a ser más responsables y obedientes, mientras que en uno de los niños se detectó signos de tristeza y aislamiento. En relación con los problemas cognitivos, el profesor de la escuela y los padres de familia reportaron falta de concentración en seis menores y cambios en el desempeño escolar en cinco de ellos. De hecho, dos infantes tuvieron dificultades para aprobar el año escolar y uno estaba repitiendo grado. Los signos psicofisiológicos más frecuentes fueron sudoración abundante (siete niños) y altos niveles de violencia hacia sus compañeros (cinco niños).

\section{Instrumento}

Para llevar a cabo la intervención se hizo una adaptación al modelo del UNICEF a partir de tres dimensiones de análisis: 1) habilidades de afrontamiento, 2) conocimiento en prevención de desastres y 3) capacidades de resiliencia.

Con base en estas dimensiones, se elaboraron dos cuestionarios con preguntas abiertas y cerradas, considerando las siguientes variables:

1. Rasgos sociodemográficos: edad, género y rasgos socioeconómicos familiares.

2. Percepción sobre el desastre: conceptualización sobre el tipo y las características del evento.
3. Tipo de afrontamiento: características y signos conductuales, emocionales y cognitivos de los infantes para manejo del riesgo y los desastres.

4. Medidas de mitigación y prevención: conocimiento, habilidades y acciones de los sujetos para mediar con los efectos de los desastres.

5. Resiliencia: habilidades para sobreponerse positivamente y desarrollar nuevos recursos para conservar la salud emocional.

Se utilizaron las mismas dimensiones y variables tanto para el pre test como para el postest, a fin de registrar los posibles cambios a partir de la intervención. Los instrumentos se administraron una semana antes del inicio del taller y una semana después de su conclusión.

\section{RESULTADOS}

En general, el pre test mostró que la totalidad de los participantes tendían a relacionar a los desastres con una experiencia triste, un accidente o una situación confusa. En cuanto al conocimiento previo sobre los riesgos y desastres, ocho de los menores se remitieron a su vivencia más próxima, es decir, las inundaciones; ninguno de ellos apuntó hacia otras posibles catástrofes que, bajo ciertas circunstancias, podían llegar a enfrentar como: los incendios, los terremotos, los tsunamis o las hambrunas.

Cuando se les preguntó sobre sus emociones y sentimientos frente al desastre, siete menores externaron haber sentido mucha tristeza, mientras que tres dijeron haber experimentado temor por su situación. Este tipo de sentimientos pueden conducir al aislamiento, provocar depresión, generar falta de concentración o acentuar la timidez (Quiroz, 2005). Si triangulamos estas respuestas con las obtenidas cuando se les cuestionó sobre la forma en que la inundación había cambiado sus vidas, vemos que los nueve niños y niñas manifestaron que después de este evento sus vidas se habían tornado fundamentalmente tristes. Entre los rasgos que se pudieron identificar estuvieron la sensación de pérdida, el miedo a que se repitiera el siniestro, la falta de orientación sobre lo que debían hacer y la incertidumbre sobre lo que pasaría después, cuando tuvieran que regresar a su vida cotidiana.

Con respecto a los valores que deben prevalecer cuando ocurre un desastre, los infantes señalaron: la 
unión familiar (dos menores), la solidaridad con los vecinos (seis menores) y la ayuda a las personas más necesitadas (un menor). Estos valores son esenciales tanto para el manejo de los desastres como para sobreponerse a ellos, es decir, para lograr la resiliencia (Henderson \& Milstein, 2003). Sin embargo, de manera paralela se pudo observar que los sujetos se asumían a sí mismos como víctimas pasivas que planteaban muy pocas alternativas para la prevención de riesgos y siniestros (Tanner, Rodríguez \& Lazcano, 2008) como: cambiar de residencia hacia otro lugar libre de anegaciones (siete niños) y buscar refugio en los albergues (tres niños).

Finalmente, se les pidió a los menores escribir una historia y hacer un dibujo en donde el tema principal fuera las inundaciones. En los relatos sobresalió un aspecto en particular: la descripción de lo ocurrido durante el desastre siempre en relación con su familia, es decir, cómo lo habían vivido, lo que pasó con sus casas, la decisión de hacia dónde ir y lo que hicieron al momento de encontrar refugio. Mientras que en los dibujos, lo más recurrente fue la presencia de nubes negras, animales muertos y el agua alrededor de las casas.

Es importante señalar que aquí no se pretendió hacer un análisis psicográfico de los dibujos o un examen estructural de los relatos; se incluyeron porque el Modelo de Recuperación Psicoafectiva del UnICEF trabaja a partir de actividades lúdico-educativas, entre las que se incluyen los dibujos y los cuentos. En la investigación, estos constituyeron una vía para conocer lo que los participantes experimentaron durante las inundaciones.

Sobre el papel del juego en las intervenciones, Garaigordobil (2005) sostiene que éste puede servir como estrategia para ayudar al niño a asimilar las experiencias que rebasan su capacidad de respuesta. Así, la utilidad del juego reside en que permite al infante “...desarrollar su pensamiento, satisfacer sus necesidades, elaborar experiencias traumáticas, descargar sus tensiones, explorar y descubrir, el goce de crear, colmar su fantasía, reproducir sus adquisiciones asimilándolas, relacionarse con los demás y ensanchar los horizontes de sí mismo..." (Garaigordobil, 1996, pág. 59).

Como se ha señalado, después de la intervención se aplicó un postest para observar si existía algún cambio en el comportamiento de los participantes. Con res- pecto a la idea original que tenían sobre los desastres, a excepción de un caso, los menores señalaron ahora que éstos podían ser vistos como una oportunidad de cambio. Además, todos fueron capaces de mencionar otros ejemplos, como los incendios, los terremotos, las enfermedades y la falta de comida.

La tristeza y el temor continuaron siendo los sentimientos más señalados, pero, en esta ocasión, ocho de los infantes dijeron que lo ocurrido también los había motivado a "hacer algo", como no tirar basura, ahorrar el agua o sembrar árboles. Una vez más, si esto se triangula con sus respuestas sobre el impacto que la inundación trajo a sus vidas, los participantes destacaron los cambios familiares, escolares e incluso materiales ocurridos después del siniestro. Esto no quiere decir que hubiesen olvidado totalmente sus experiencias negativas, pero sí fueron capaces de mirar otras dimensiones del evento, por ejemplo, dos niñas comentaron sobre su promoción al siguiente grado escolar, tres niñas y dos niños hablaron sobre las obras de remodelación que sus padres habían hecho para protegerse del agua y otros dos dijeron que no habían tenido cambios significativos.

Los resultados del postest ofrecieron varias pistas sobre los aprendizajes en el manejo de desastres. Las respuestas en ese sentido fueron: estar alerta a las indicaciones de protección civil y buscar el refugio más cercano (dos niños), así como permanecer con la familia e identificar las rutas de evacuación (ocho niños). Aquí se puede observar una diferencia importante con respecto al pre test, en donde ninguno de los participantes acertó a mencionar alguna de las medidas que se deben seguir en estos casos.

En contraste, las acciones de prevención y los valores no mostraron variación entre el pre test y el postest. Los menores contestaron prácticamente lo mismo en ambos instrumentos. Finalmente, están los resultados de la historieta y el dibujo. En esta ocasión, los participantes abarcaron cuestiones que estuvieron ausentes en el pre test, como la preocupación por el deterioro ambiental, una presencia más protagónica de cada uno de los integrantes de la familia y el interés por los animales que murieron por el siniestro. En los dibujos volvieron a plasmar algunas de las dificultades que enfrentaron, como sus casas rodeadas de agua, pero a la par, se incorporaron algunos señalamientos para cui- 
dar el medio ambiente como: cerrar las llaves, no tirar basura y plantar árboles.

\section{DISCUSIÓN}

El propósito principal de una intervención psicológica como la que se realizó en esta investigación, es ayudar a los menores a que superen algunas de las secuelas que a nivel psicoafectivo pudieron haberse provocado después de un evento de gran magnitud, como las inundaciones. Los beneficios en la salud emocional dependen de varios factores, como la intensidad del fenómeno, el tiempo que se estuvo expuesto al peligro, el conocimiento previo sobre su manejo y el apoyo familiar, entre otros (Gerrity \& Flinn, 2000).

En el caso que se expuso, además, tuvimos otras variables que sin duda pudieron haber intervenido en los efectos del modelo, una de ellas fue la vulnerabilidad de la propia comunidad, no sólo porque sus habitantes han sufrido anegaciones constantemente, sino porque existen condiciones sociales poco favorables. Como señalan Rodríguez y González (2007), la recuperación psicoafectiva puede verse limitada por problemas estructurales, como la pobreza, la marginación y la violencia, todas ellas condiciones que se cumplían en nuestros sujetos de estudio. Así, aunque se trabaje preventiva y correctivamente, recurriendo a diferentes estrategias psicológicas, en términos reales, el modelo sólo puede influir en una de las muchas dimensiones que están implicadas en un desastre.

Adicionalmente, cuando se lleva a cabo una intervención se sugiere profundizar en las manifestaciones psicológicas de los sujetos y, si es posible, en las de la comunidad en general (García, Gil \& Valero, 2007). En esta investigación no fue posible llegar a ese nivel debido a la disponibilidad de los propios menores y a la limitada participación de sus padres y profesores.

Aunado a esto, el trabajo sólo pudo realizarse con un pequeño grupo de niños afectados, lo que plantea dificultades para generalizar los resultados. En ese sentido, la investigación se asemeja a la desarrollada por Santiesteban, Castro, González y Sánchez (2010), en la que también se abordó a una muestra reducida de infantes. No obstante, este tipo de ejercicios puede servir para hacer ajustes y, como proponen los autores, implementarlos después a gran escala. Una vez hecho esto, entonces, se puede empezar a planear la siguiente etapa, que es la del diseño de políticas para la atención a la salud mental de los niños y niñas.

El modelo en general, tal como lo formula el UNICEF, también tiene ciertas salvedades. En primer lugar porque se trata de una intervención para la recuperación, esto es, sólo se puede utilizar una vez que ha ocurrido el siniestro, por lo que se necesitan más propuestas orientadas hacia la prevención y con una perspectiva nacional o local. En segundo lugar, porque se carece de una metodología de evaluación, con parámetros o indicadores estandarizados, que permita valorar la confiabilidad de los resultados. Finalmente, porque hacen falta más trabajos académicos que analicen de manera crítica los beneficios logrados en diferentes situaciones de desastre y contextos geográficos. Hasta ahora, predominan los manuales y reportes de intervenciones in situ, lo que disminuye las posibilidades de hacer comparaciones que se traduzcan en mejoras para el propio modelo.

\section{CONCLUSIONES}

La intervención con este grupo de niños y niñas afectados por las inundaciones permitió visualizar algunas líneas y temáticas que podrían ser trabajadas con poblaciones más grandes y a largo plazo. Una de ellas es la ampliación del rol de víctima ante los desastres (Tanner, Rodríguez \& Lazcano, 2008), para lo cual, se requiere fortalecer los conocimientos previos de los sujetos y ayudarlos a distinguir las oportunidades que este tipo de situaciones les presentan. Otra cuestión es la participación de los agentes que pueden contribuir al desarrollo de contextos y habilidades resilientes, como los padres y maestros (Henderson \& Milstein, 2003). Por último, está el tema del peso que tienen otros factores, sociales y psicológicos, en la efectividad y permanencia de los resultados de la intervención. • 


\section{REFERENCIAS}

Caswell, A. (2009). Guía básica de atención a la niñez en emergencias: El acompañamiento psicoemocional. México: Save the children México.

CEPAL (2011). Tabasco: características e impacto socioeconómico de las lluvias extremas de 2008. México: Comisión Económica para América Latina y el Caribe.

CEPAL (2012). Impacto socioeconómico de las inundaciones registradas en el estado de Tabasco de septiembre a noviembre de 2011. México: Comisión Económica para América Latina y el Caribe.

García, M., Gil, M. \& Valero, M. (2007). Psicología y desastres: aspectos sociales. Castellón de la Plana: Universitat Jaume I.

Garaigordobil, M. (1996). Evaluación de una intervención psicoeducativa en sus efectos sobre la conducta prosocial y la creatividad. Madrid: Ministerio de Educación y CulturaCentro de Investigación y Documentación Educativa.

Garaigordobil, M. (2005). Diseño y evaluación de un programa de intervención socioemocional para promover la conducta prosocial y prevenir la violencia. Madrid: Ministerio de Educación y Cultura-Centro de Investigación y Documentación Educativa.

Gerrity, E. \& Flinn, B. (2000). Consecuencias de los desastres en la salud mental, en E. Noji (Ed.). Impacto de los desastres en la salud pública (pp. 101-121). Bogotá: Organización Panamericana de la Salud.

Henderson, N. \& Milstein, M. (2003). Resiliencia en la escuela. Buenos Aires: Paidós.

Muñoz, V. \& De Pedro, F. (2005). Educar para la resiliencia. Un cambio de mirada en la prevención de situaciones de riesgo social. Revista complutense de educación, 16(1), 107-124.

Palacio, M. (2000). Promoción de la salud y prevención de la enfermedad de los niños en emergencias complejas o situaciones de desastre. Bogtá: Organización Panamericana de la Salud. Bogotá.

Quiroz, N. (2005). Recuperación psicoafectiva de niños afectados por desastres y conflicto armado. Colombia: Fondo de las Naciones Unidas para la Infancia. 35 p.

Robles, J. \& Medina, J. (2002). Intervención psicológica en las catástrofes. Madrid: Síntesis.

Rodríguez, J. \& González, R. (2007). La reforma de los servicios de salud mental: 15 años después de la Declaración de Caracas. Washington: Organización Panamericana de la Salud.
Santiesteban, Y., Castro, M., González, Z. \& Sánchez, L. (2010). Impacto de los huracanes Gustav e Ike en la salud psicológica de un grupo de escolares afectados. Revista cubana de medicina general integral, 26(3), http://bvs.sld.cu/revistas/ mgi/vol_26_3_10/mgi08310.htm

Save the Children (2002). Manual para la prevención y atención a niños y niñas y adolescentes ante desastres. Managua: Save the Children México.

Save the Children (2006). Plan de prevención y atención de desastres. México: Save the Children México.

Tanner, T., Rodríguez, G. \& Lazcano, J. (2008). Los niños y niñas y la gestión de riesgo: Un rol clave para la prevención de desastres. Medio ambiente y urbanización, 69(1), 117-134.

Taylor, A. (1989). Disasters and disaster stress. Nueva York: AMS Press.

UNEP (2007). Environment and disaster risk. Emerging perspectives. Ginebra: United Nations Environment Program.

UNICEF (2003). Aprendamos a prevenir los desastres. ¡Los niños y las niñas también participamos en la reducción de riesgos! San José de Costa Rica: Fondo de las Naciones Unidas para la Infancia.

UNICEF (2005). Manual para situaciones de emergencias sobre el terreno. Nueva York: Fondo de las Naciones Unidas para la Infancia.

UNICEF (2007). El retorno a la alegría. Manual de protocolos y juegos para terapias lúdicas. República Dominicana: Fondo de las Naciones Unidas para la Infancia.

UNICEF (2008a). Albergues en escuela, ¿cuándo?, ¿cómo?, ¿por $q u e ́$ ? Ginebra: Fondo de las Naciones Unidas para la Infancia.

UNICEF (2008b). El retorno a la alegría. Resultados preliminares y proyecciones. República Dominicana: Fondo de las Naciones Unidas para la Infancia.

UNICEF (2009). Educación en emergencias: cómo incluir a todos. Ginebra: Fondo de las Naciones Unidas para la Infancia Inter-Agency Network for Education in Emergencies.

UNICEF (2010). El retorno a la alegría. Manual de capacitación. República Dominicana: Fondo de las Naciones Unidas para la Infancia.

Uriarte, J. (2005). La resiliencia. Una nueva perspectiva en psicopatología del desarrollo. Revista psicodidáctica, 10(2), 61 - 79.

Fecha de recepción: junio 2012 Fecha de aceptación: noviembre 2012 